\title{
FICOCIANINA COMO INDICADOR DE CIANOBACTÉRIAS EM RESERVATÓRIOS
}

\author{
Thiago T. da Silva ${ }^{a, *,(}$, Rufina A. M. Albuquerque ${ }^{a}$ e Helena Becker ${ }^{a}$ \\ aDepartamento de Química Analítica e Físico Química, Universidade Federal do Ceará, 60455-760 Fortaleza - CE, Brasil
}

Recebido em 12/04/2021; aceito em 22/07/2021; publicado na web em 27/08/2021

\begin{abstract}
PHYCOCYANIN AS AN INDICATOR OF CYANBACTERIA IN RESERVOIRS. Phycocyanin is a pigment present in cyanobacteria whose optical characteristics favor its application in clinical, dietary, and environmental aspects. There are reports of studies of these pigments in lakes and reservoirs, which present different analysis methodologies. Based on this information, this work aims to evaluate correlations between phycocyanin and the number of cyanobacteria to optime the methodologies mentioned above. The samples were collected from four reservoirs in the semiarid region of Ceará, and the applied methodology was based on the work of Hórvath (2013). The spectrophotometry and the fluorimetry analyzes were performed in this analysis. The analytical curves for the phycocyanin showed high linearity, and the fluorimetric technique, which was used due to its greater analytical sensitivity. The absorbance coefficient $\left(2.57 \mathrm{~mL} \mathrm{mg}^{-1} \mathrm{~cm}^{-1}\right)$ was obtained by spectrophotometry, which was lower than that reported in the literature. The correlations found were higher and significant for phycocyanin $(r>0.70)$ for the reservoirs, while the correlations with chlorophyll were much lower, hence is contrary to what was observed in the literature. It is concluded that the phycocyanin pigment should be used as an indicator in the estimation of cyanobacteria in reservoirs, replacing the use of chlorophyll for the same function.
\end{abstract}

Keywords: monitoring; analytical curve; correlation; semiarid.

\section{INTRODUÇÃO}

As ficobilinas são substâncias que auxiliam nos processos fotossintéticos realizados por alguns grupos de organismos aquáticos, como algas e cianobactérias, sendo classificadas como "pigmentos acessórios". Apresentam características fluorescentes e são solúveis em água, presentes em cianobactérias, dinoflagelados, criptofíceas e rodófitas. ${ }^{1,2}$ Essas ficobilinas são produzidas em larga escala por esses microrganismos e podem compor até $50 \%$ das proteínas celulares solúveis. Elas são compostas pelas seguintes classes de proteínas: ficocianina, ficoeritrina e aloficocianina, que apresentam diferentes regiões de absorção máxima espectral e diferentes origens nos organismos citados. , $^{1,3,4}$

A aloficocianina, ficoeritrina e ficocianina apresentam, respectivamente, absorção na região de $650-655 \mathrm{~nm}, 540$ - $570 \mathrm{~nm}$ e $610-620 \mathrm{~nm} .^{2,5,6}$ Em estudos comparativos realizados por Patel et al., sobre a quantidade dos pigmentos presentes em cianobactérias (exceto clorofila $a$ ), foi constatado que a quantidade do pigmento ficocianina era muito superior (acima de 75\%) em relação aos outros pigmentos. ${ }^{7}$ Devido a isso, estudo das cianobactérias a partir de análises desse pigmento apresenta potencial viabilidade para o monitoramento desses organismos em água. ${ }^{8,9}$

O monitoramento do desenvolvimento e proliferação das cianobactérias em águas continentais é uma ação relevante, devido à liberação de toxinas por algumas espécies deste grupo de microrganismos. Essas toxinas, acima de determinadas concentrações, podem causar efeitos dermatotóxicos, neurotóxicos e hepatotóxicos aos seres vivos. ${ }^{10,11}$

Com frequência, encontram-se publicações relatando também a clorofila a como um parâmetro para estimativa de biomassa fitoplanctônica. ${ }^{6,12-15}$ Esse pigmento encontra-se presente em todos os organismos fitoplanctônicos e, em alguns casos (principalmente em condições de concentração elevada), não apresenta confiabilidade na estimativa de uma elevada densidade de cianobactérias encontrada nos corpos hídricos.

*e-mail: thiagorrmf@yahoo.com.br
A ficocianina pode ser subdividida em dois tipos, de acordo com a origem desse pigmento nos microrganismos: C-Ficocianina (oriunda de cianobactérias) e R-Ficocianina (oriunda de algas vermelhas/ rodófitas), sendo as rodófitas praticamente exclusivas de ambientes marinhos, enquanto as cianobactérias são abundantes em ambientes de água doce..$^{4,16}$

No aspecto clínico, a ficocianina tem sido apontada em estudos como um antioxidante eficiente e como um anti-inflamatório. ${ }^{17,18}$ Além disso, possui aplicações como marcador biomédico em pesquisas. ${ }^{19}$ No aspecto comercial, esse pigmento é utilizado em alimentos (sorvetes, doces e refrescos), alem da utilização em cosméticos. ${ }^{20}$ Por apresentar certas propriedades, como fluorescência e solubilidade em água, pode ser usada como indicador da presença de cianobactérias nos corpos hídricos. ${ }^{8,21,22}$

$\mathrm{Na}$ estrutura das cianobactérias, a ficocianina está localizada no hialoplasma, sendo necessário o rompimento de sua parede celular para disponibilizar o pigmento no meio e realizar a análise da solução. ${ }^{22}$ Para o processo de ruptura celular, diversos procedimentos são relatados, como tratamento enzimático, congelamento/ descongelamento e ruptura sônica.

$\mathrm{Na}$ literatura há diferentes técnicas analíticas para análise da ficocianina em água. Algumas delas são apresentadas na Tabela 1.

Tabela 1. Técnicas de análise para ficocianina

\begin{tabular}{ll}
\hline Técnica & \multicolumn{1}{c}{ Autores (ano publicação) } \\
\hline CLAE & $\begin{array}{l}\text { Saluri, Kaldmäe, Tuvikene (2019); Viskari, Colyer } \\
(2003)^{6,23}\end{array}$ \\
\hline Eletroforese & Boussiba, Richmon (1979); Soni et al. $(2006)^{24,25}$ \\
\hline $\begin{array}{l}\text { Fotometria } \\
\text { (UV-VIS) }\end{array}$ & $\begin{array}{l}\text { Bennett, Bogorad (1973); Lawrenz } \text { et al. }(\text { 2011); Sarada } \\
\text { et al. (1999); Silveira } \text { et al. (2007); Tsola } \text { et al. } \text { (2018) })^{26-30}\end{array}$ \\
\hline Fluorimetria & $\begin{array}{l}\text { Gregor } \text { et al. (2007); Lehman (2007); Pavlac } \text { et al. } \text { (2012); } \\
\text { Seppala } \text { et al. (2007); Stewart, Farmer (1984); Thomson- } \\
\text { Laing, Puddick, Wood (2020) })^{8,31-35}\end{array}$ \\
\hline
\end{tabular}

Alguns trabalhos apresentam diferentes expressões para a quantificação da ficocianina em amostras ou padrões, com 
adaptações e/ou correções em outras expressões amplamente utilizadas, como nas publicações de Yéprémian et al. e Nair et al. ${ }^{4,36}$ O trabalho desenvolvido por Bennett e Bogorad em 1973, intitulado Complementary chromatic adaptation in a filamentous blue-green alga é um dos mais citados na literatura associada às cianobactérias. ${ }^{26}$ Esse estudo apresenta expressões matemáticas para o cálculo das concentrações das ficobilinas, sendo utilizadas em diversos trabalhos para quantificação desses pigmentos. A expressão que calcula a concentração da ficocianina é apresentada na equação 1 .

$$
\mathrm{C}-\mathrm{FC}=\frac{\mathrm{ABS}_{615}-0,474 \cdot \mathrm{ABS}_{652}}{5,34}
$$

sendo: C-FC = concentração de ficocianina $\left(\mathrm{mg} \mathrm{mL}^{-1}\right) ; \mathrm{ABS}_{615} \mathrm{e}$ $\mathrm{ABS}_{652}=$ valor da absorbância nos comprimentos de onda de 615 e 652 , respectivamente.

Apesar de ser amplamente utilizada na quantificação desse pigmento em diversos trabalhos científicos, a expressão anterior foi elaborada em condições específicas de análises das ficobilinas, da forma descrita por seus autores. Essas condições não são sempre observadas na descrição metodológica de alguns trabalhos. ${ }^{21,22,28}$

Diante das informações expostas, este trabalho tem como objetivo analisar o pigmento ficocianina em amostras de reservatórios, apresentando uma otimização das metodologias descritas, visando a avaliar as possíveis correlações da ficocianina com o número de cianobactérias presentes em águas de reservatórios do semiárido cearense.

\section{PARTE EXPERIMENTAL}

\section{Área de estudo}

Os reservatórios estudados foram Gavião (em Pacatuba), Riachão (em Itaitinga), Pacoti (em Horizonte) e Pacajus (em Pacajus), presentes na Região Metropolitana de Fortaleza/CE. A Figura 1 ilustra a localização de cada um desses reservatórios.
Os reservatórios estão localizados na região semiárida do país, que apresenta elevadas temperaturas e baixa pluviosidade durante o ano, principalmente durante o segundo semestre, ocasionando situações de déficit hídrico e piora da qualidade/estado trófico das águas armazenadas. ${ }^{37}$

As coletas foram realizadas por técnicos da Companhia de Gestão dos Recursos Hídricos do Ceará (COGERH), responsável pelo gerenciamento dos reservatórios cearenses. As amostras de água foram coletadas em um ponto próximo a barragem, à profundidade de 0,3 metros, sendo armazenadas em frascos escuros, identificadas e acondicionadas em ambiente refrigerado até a realização das análises. Os dados obtidos são referentes à análise mensal nesses reservatórios, durante o período de 2017 a 2019, perfazendo 36 amostras para cada reservatório.

\section{Reagentes e vidrarias}

As soluções foram preparadas utilizando água ultrapura (Milli-Q) e reagentes de grau analítico (PA). Na elaboração da solução estoque de ficocianina, foi utilizado o reagente C-Phycocyanin (Sigma-Aldrich - Referência 52468), cuja concentração indicada era $\geq 10,0 \mathrm{mg} \mathrm{mL}^{-1}$.

As vidrarias utilizadas nos experimentos foram materiais comuns de laboratório (balão volumétrico, béquer, erlenmeyer, entre outros), todos descontaminados com solução de $\mathrm{HCl} 5 \%$.

\section{Análise de ficocianina}

Determinação da concentração do reagente padrão e elaboração da curva analítica de ficocianina

Para determinação da concentração do reagente C-Phycocyanin, foram recolhidos $10 \mu \mathrm{L}$ e transferidos para um balão de $10 \mathrm{~mL}$, sendo aferido com solução tampão salino $\left(0,01 \mathrm{~mol} \mathrm{~L}^{-1}\right.$ de fosfato de sódio $+0,15 \mathrm{~mol} \mathrm{~L}^{-1}$ de $\mathrm{NaCl}$ ). Foi realizada varredura da solução aferida utilizando Espectrofotômetro UV-Vis Marca Varian modelo Cary $1 \mathrm{E}$ e calculado a concentração segundo Bennett e Bogorad. ${ }^{26}$
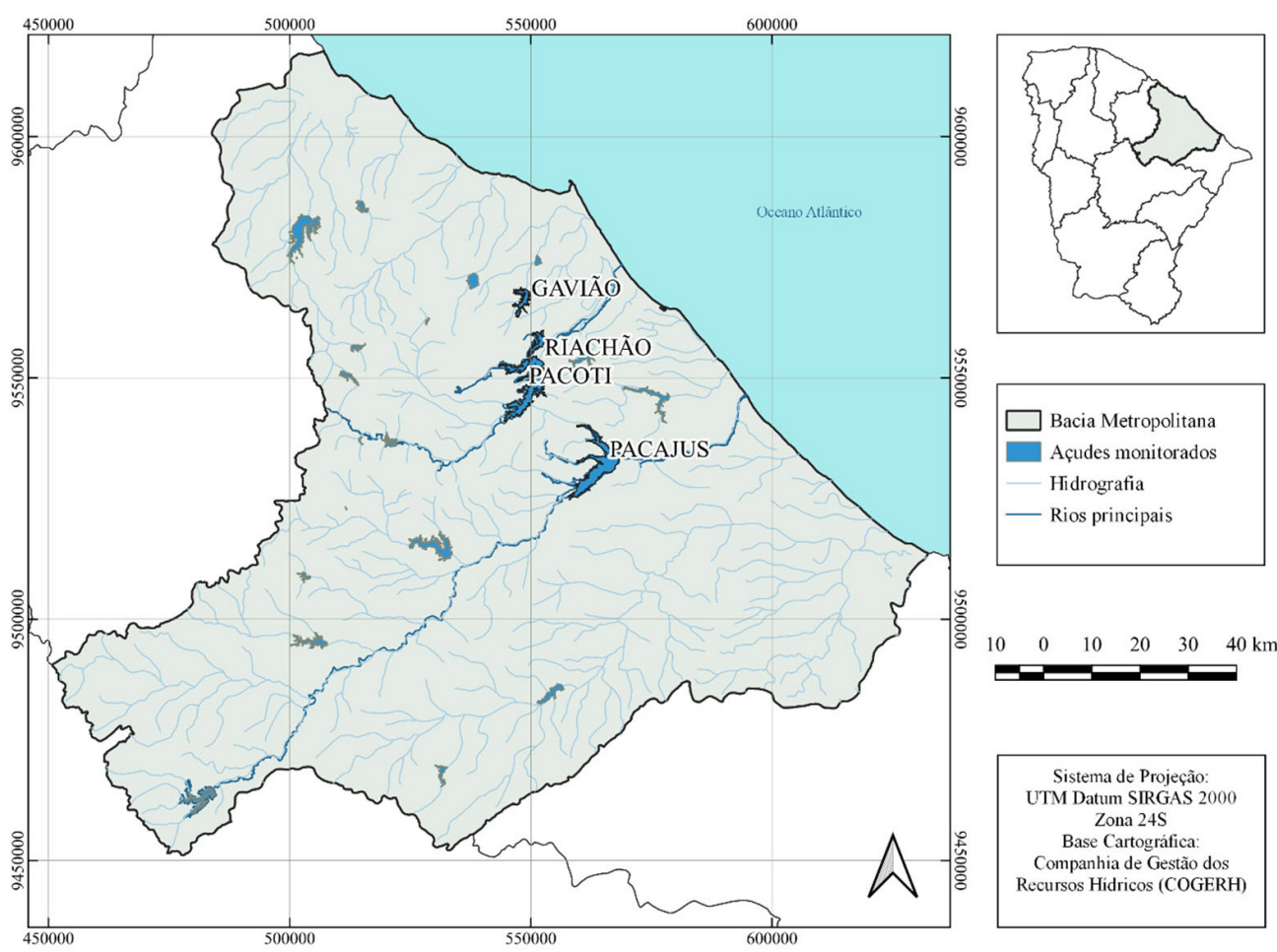

Sistema de Projeção: UTM Datum SIRGAS 2000 Zona $24 S$ Base Cartográfica Companhia de Gestão dos Recursos Hidricos (COGERH)

Figura 1. Mapa dos reservatórios estudados da Bacia Metropolitana 
Na elaboração da solução estoque da curva de ficocianina foram recolhidos $40 \mu \mathrm{L}$ do reagente $\mathrm{C}$-Phycocyanin e transferidos a uma balão de $10 \mathrm{~mL}$, sendo aferido com solução tampão de fosfato $\mathrm{pH} 7,2$ $\left(0,025 \mathrm{~mol} \mathrm{~L}^{-1}\right)$. As soluções subsequentes da curva foram preparadas por diluição de determinados volumes retirados da solução estoque, todos sendo transferidos para balões volumétricos de $5 \mathrm{~mL}$ e aferidos com tampão de fosfato. A solução tampão de fosfato preparada foi utilizada na determinação do branco do análise. As soluções foram analisadas por espectrofotometria $(\lambda=619 \mathrm{~nm})$ e fluorimetria $(\lambda=644 \mathrm{~nm}$ ) (aparelho da Shimadzu modelo RF-6000), usando cubeta de quartzo de $1 \mathrm{~cm}$ de caminho óptico para ambas situações.

O limite de detecção (LD) e o limite de quantificação (LQ) para a análise foram calculados utilizando as equações da reta (em triplicata) da curva analítica, de acordo com a indicação da Agência Nacional de Vigilância Sanitária (ANVISA). ${ }^{38}$

$$
\begin{aligned}
& \mathrm{LD}=\frac{3,3 \sigma}{\mathrm{IC}} \\
& \mathrm{LQ}=\frac{10 \sigma}{\mathrm{IC}}
\end{aligned}
$$

sendo: $\sigma=$ desvio padrão dos coeficientes lineares e $\mathrm{IC}=$ média dos coeficientes angulares.

\section{Análise de ficocianina nas amostras}

O procedimento aqui descrito é semelhante ao exposto no trabalho de Hórvath et al., que contém o processo de congelamento e descongelamento da amostra, seguido de sonicação. ${ }^{21} \mathrm{~A}$ sequência do procedimento é apresentada pelo fluxograma da Figura 2.

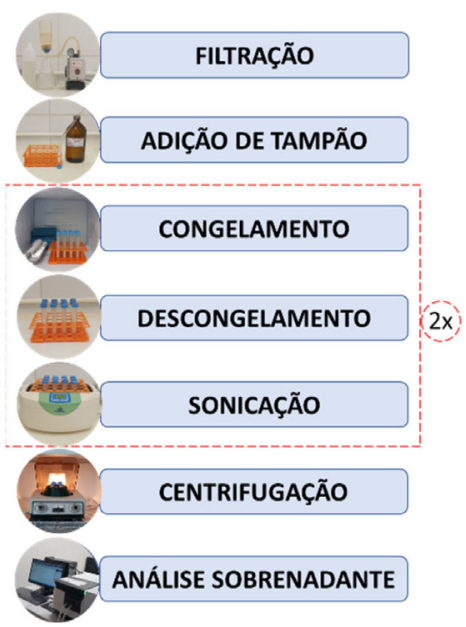

Figura 2. Fluxograma das etapas de análise da ficocianina

As amostras foram filtradas por um sistema de filtração, composto por: bomba à vácuo, kitassato, papel de filtro e copo volumétrico de filtração. Volume de $200 \mathrm{~mL}$ foi filtrado (em duplicata) utilizando filtros de fibra de vidro da marca Macherey-Nagel (GF-3; poros de $0,6 \mu \mathrm{m})$. Após a filtração, os filtros foram colocados em tubos de Falcon.

Foram adicionados $15 \mathrm{~mL}$ da solução tampão de fosfato $\mathrm{pH} 7,2$ aos tubos de Falcon que continham os filtros das amostras, sendo agitados em seguida. As amostras foram armazenadas em congelador $\left(-18^{\circ} \mathrm{C}\right)$ em torno de $4 \mathrm{~h}$, sendo retiradas após o referido tempo para posterior descongelamento.

No processo de sonicação foi utilizado aparelho Ultrassom marca Cristófoli durante 180 segundos $(42 \mathrm{kHz})$. As etapas de "freezing/ thawing/sonication" foram realizadas duas vezes.
Após a sonicação, elas foram centrifugadas (centrífuga Hettich EBA 8S) durante 15 minutos a $4000 \mathrm{rpm}$, havendo controle de temperatura $\left(9,0 \pm 0,1^{\circ} \mathrm{C}\right)$ no interior da incubadora (marca Marconi modelo B.O.D MA 415). Finalizado o processo, os sobrenadantes das amostras foram analisados por espectrofluorimetria.

\section{Análise de clorofila $a$ e cianobactérias}

A quantificação do pigmento clorofila $a$ - expressa em $\mu \mathrm{g} \mathrm{L}^{-1}$ - foi feita por espectrofotometria (método $10200 \mathrm{H}$ ), utilizando solução de acetona $90 \%$ e solução ácida na quantificação de feofitina, realizada pela Companhia de Água e Esgoto do Ceará (Cagece). ${ }^{39} \mathrm{~A}$ determinação de cianobactérias nas amostras (expressa em cél $\mathrm{mL}^{-1}$ ) foi feita pela metodologia de Utermöhl, realizadas pelo Instituto de Tecnologia de Pernambuco (ITEP), que presta serviços à COGERH. ${ }^{40}$

\section{Correlação entre parâmetros}

O coeficiente de correlação de Pearson (r) foi utilizado na avaliação das correlações obtidas nesse estudo. A "força" dessas associações entre os parâmetros foi definida pelas faixas apresentadas na Tabela $2{ }^{41}$

Tabela 2. "Força" das correlações de Pearson (r)

\begin{tabular}{cc}
\hline $\begin{array}{c}\text { Faixa de } \mathrm{r} \\
(\text { em módulo })\end{array}$ & Classificação \\
\hline $0,0 \leq|\mathrm{r}| \leq 0,2$ & Não-correlacionado \\
$0,2<|\mathrm{r}| \leq 0,4$ & Correlação fraca \\
$0,4<|\mathrm{r}| \leq 0,6$ & Correlação moderada \\
$0,6<|\mathrm{r}| \leq 0,8$ & Correlação forte \\
$0,8<|\mathrm{r}| \leq 1,0$ & Correlação muito forte \\
\hline
\end{tabular}

\section{RESULTADOS E DISCUSSÃO}

\section{Quantificação da ficocianina}

Utilizando a equação 1 , a concentração calculada do padrão de ficocianina foi $16,1 \mathrm{mg} \mathrm{mL}^{-1}$, valor esse em concordância com a informação presente no rótulo do reagente. A partir dessa informação de concentração, as soluções que compõem a curva analítica foram elaboradas da faixa de 2,66 a 44,40 $\mathrm{mg} \mathrm{L}^{-1}$, apresentadas na Figura 3.

As equações das retas calculadas apresentam ótima tendência de linearidade, porém, a equação proveniente da técnica de fluorimetria apresenta maior sensibilidade analítica (coeficiente angular), sendo ela utilizada na quantificação da ficocianina nas amostras dos reservatórios. A equação final utilizada na quantificação de ficocianina é indicada na equação 4.

$$
\mathrm{C}-\mathrm{FC}=\left(\frac{\mathrm{I}_{644}-11,608}{43,249}\right) \times 0,075
$$

sendo: $\mathrm{I}_{644}=$ intensidade fluorimétrica em $644 \mathrm{~nm}$ e 0,075 = fator de correção da solução (volume de tampão utilizado - $15 \mathrm{~mL}$ / volume de amostra filtrado - $200 \mathrm{~mL}$ ).

A concentração da ficocianina calculada por fluorimetria variou de 1,21 à $17,95 \mathrm{mg} \mathrm{L}^{-1}$ nos reservatórios. O limite de detecção (LD) e o limite de quantificação (LQ) foram de $0,36 \mathrm{mg} \mathrm{L}^{-1}$ e $1,19 \mathrm{mg} \mathrm{L}^{-1}$, respectivamente.

Pela equação da reta realizada por espectrofotometria, obtémse o coeficiente de extinção da ficocianina por esse método de análise $\left(2,57 \mathrm{~mL} \mathrm{mg}^{-1} \mathrm{~cm}^{-1}\right)$, valor inferior ao presente no trabalho 

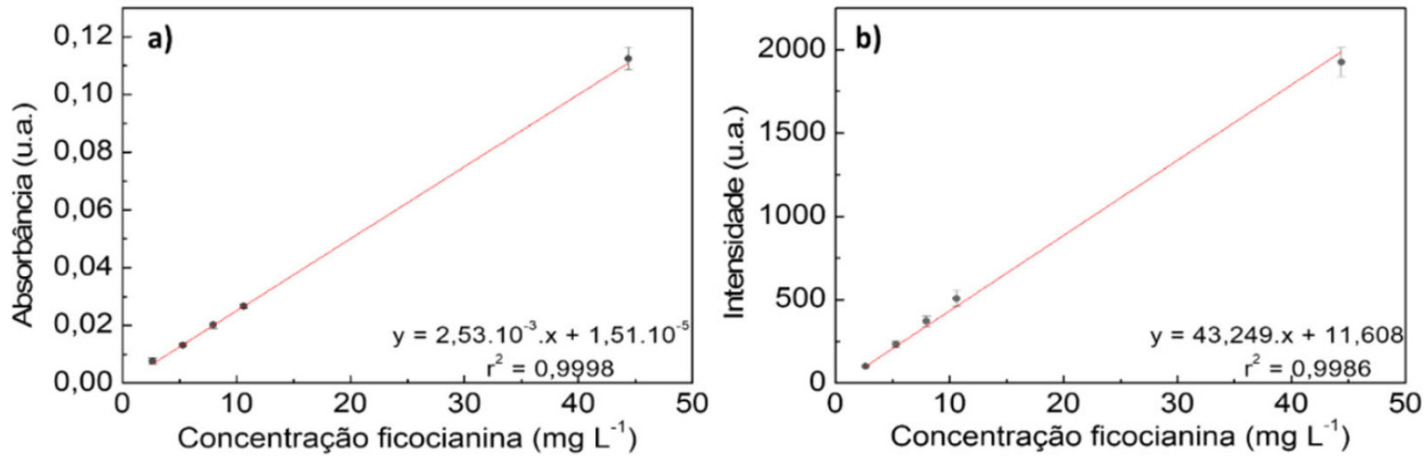

Figura 3. Equação da reta para as técnicas: espectrofotometria (a) e fluorimetria (b)

de Bennett e Bogorad. ${ }^{26}$ A diferença desses valores de coeficientes, também relatado em outros trabalhos científicos (Tabela 3), pode estar associada ao $\mathrm{pH}$ e força iônica das soluções utilizadas nas etapas de extração. ${ }^{42}$

\section{Cianobactérias e correlação com ficocianina}

Pelos dados fornecidos pela COGERH, os resultados de cianobactérias nos reservatórios foram da faixa de 5.000 à $600.000 \mathrm{cel} \mathrm{mL}^{-1}$, apresentando uma grande amplitude nos valores. A Figura 4 apresenta as correlações entre a ficocianina e contagem de cianobactérias para cada reservatório estudado. Os valores do coeficiente de correlação indicam boa tendência de correlação entre essas variáveis, por apresentarem valores classificados como correlação forte ou muito forte. Outras publicações científicas também apresentaram boas correlações de ficocianina e cianobactérias (contagem ou biomassa), como nos trabalhos de Hórvath $(r=0,97)$, Tsola $(r=0,84)$ e Brient $(r=0,73) .^{21,30,46}$

A literatura apresenta fatores que podem interferir na quantificação da ficocianina em água, como $\mathrm{pH}$ (valores externos a faixa 5,0-7,5), temperatura (superior a $30^{\circ} \mathrm{C}$ ), elevada luminosidade e presença e metais, sendo relatados também condições de estresse para desenvolvimento das cianobactérias. ${ }^{16,28}$

Por dados do ITEP, mais de $90 \%$ dos microrganismos

Tabela 3. Comparação bibliográfica de absortividade da ficocianina

\begin{tabular}{ccc}
\hline Absortividade $(\varepsilon)$ & Comprimento de onda $(\lambda)$ & Solução tampão \\
\hline $5,18 \mathrm{~mL} \mathrm{mg}^{-1} \mathrm{~cm}^{-1}$ & $620 \mathrm{~nm}$ & Tampão fosfato de sódio 0,1 M (pH 7) \\
$5,92 \mathrm{~mL} \mathrm{mg}^{-1} \mathrm{~cm}^{-1}$ & $615 \mathrm{~nm}$ & Tampão fosfato de sódio 0,01 M (pH 7) + NaCl 0,15 M \\
$7,2 \mathrm{~mL} \mathrm{mg}^{-1} \mathrm{~cm}^{-1}$ & $620 \mathrm{~nm}$ & Tampão fosfato 0,1 M (pH 6) \\
$7,3 \mathrm{~mL} \mathrm{mg}^{-1} \mathrm{~cm}^{-1}$ & $620 \mathrm{~nm}$ & Bennett $^{26}$ \\
$3,93 \mathrm{~mL} \mathrm{mg}^{-1} \mathrm{~cm}^{-1}$ & $618 \mathrm{~nm}$ & Tampão fosfato 0,1 M (pH 7) + lisozima + EDTA \\
$2,57 \mathrm{~mL} \mathrm{mg}^{-1} \mathrm{~cm}^{-1}$ & $619 \mathrm{~nm}$ & Tampão fosfato 0,1 M (pH 5,9) + EDTA 0,1 mM \\
\hline
\end{tabular}
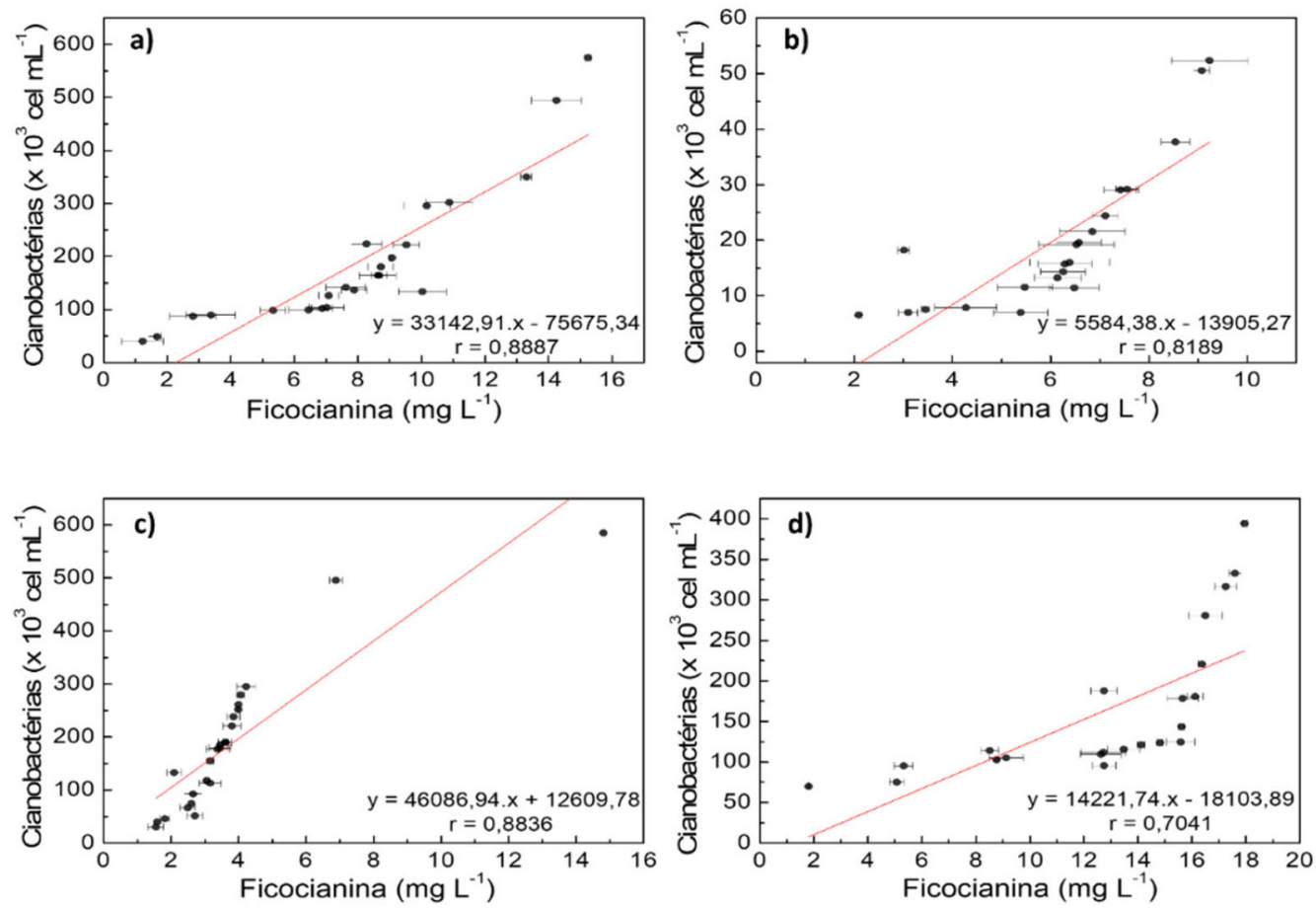

Figura 4. Correlação de ficocianina e contagem de cianobactérias nos reservatórios: Gavião (a); Pacajus (b); Pacoti (c) e Riachão (d) 

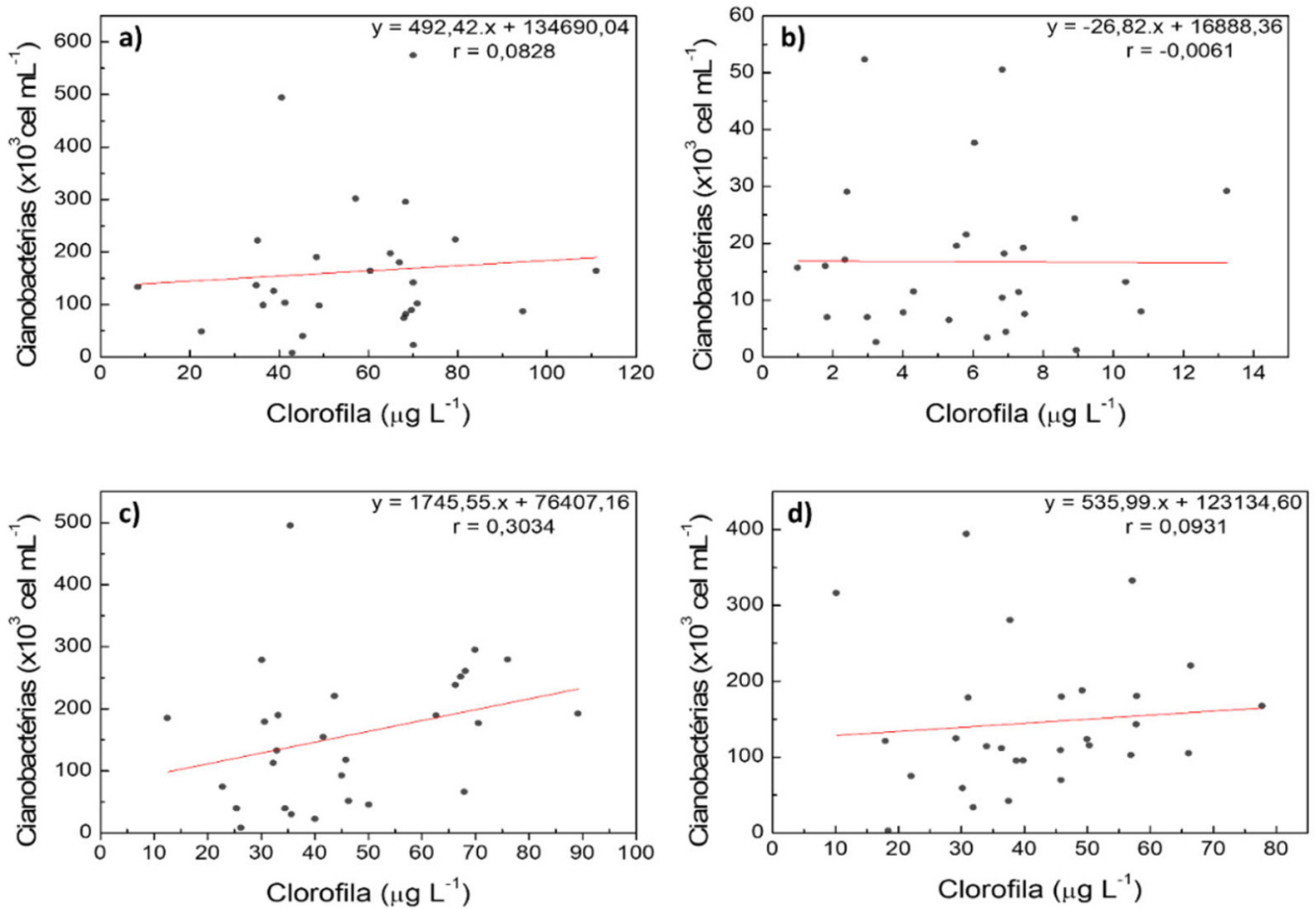

Figura 5. Correlação de clorofila a e contagem de cianobactérias nos reservatórios: Gavião (a); Pacajus (b); Pacoti (c) e Riachão (d)

identificados nas análises das amostras são pertencentes ao grupo de cianobatérias, sendo o percentual restante pertencente a outros grupos, como clorófitas e criptófitas.

Excetuando-se alguns resultados no reservatório Pacajus, os demais reservatórios apresentaram, predominantemente, valores de cianobactérias superiores ao valor máximo permitido (VMP) pela legislação brasileira vigente, segundo Resolução CONAMA 357/05 $\left(\mathrm{VMP}=50.000 \mathrm{cel} \mathrm{mL}^{-1}\right) .^{47}$

Pelos resultados de clorofila $a$ e cianobactérias, as correlações apresentaram valores bem baixos para o mesmo período de estudo, como disposto na Figura 5.

Nestes reservatórios, as correlações entre a concentração de clorofila $a$ e cianobactérias não foram significativas, apresentando o r de Pearson variando entre -0,0061 e 0,3034, condição diferente de outros resultados na literatura, como nos estudos de Izydorczyk $(\mathrm{r}=0,679)$ e Chaffin $(\mathrm{r}=0,929) \cdot{ }^{13,15}$ Os resultados sugerem a não-correspondência desses dois parâmetros nos reservatórios do semiárido cearense (correlações inexistentes ou fracas), contribuindo com indícios para busca de outra forma de correlação com cianobactérias, como os dados de ficocianina já mostrados.

As condições hidroclimáticas da região semiárida podem afetar o crescimento e desenvolvimento das cianobactérias, apresentando variadas concentrações de clorofila $a$ e influenciando nessa nãocorrespondência. Diversos fatores interferem na quantificação desses dois parâmetros, sendo a clorofila $a$ afetada por luminosidade, alterações enzimáticas, temperatura e $\mathrm{pH} .{ }^{48}$ Quanto às cianobactérias, a idade desses microrganismos, tamanho e espécie influenciam nas quantidades produzidas dos pigmentos presentes, como a clorofila $a{ }^{46,49}$

A quantificação de clorofila $a$ engloba a concentração desse pigmento oriundo de diversos produtores primários presentes nos corpos hídricos além das cianobactérias, enquanto a medida do pigmento ficocianina está intrinsicamente associada a presença de cianobactérias (embora não seja exclusiva desses microrganismos), como reafirmado pelas correlações apresentadas.

Nas duas correlações apresentadas, o reservatório Pacajus apresentou os menores valores para cianobactérias e, na correlação com clorofila $a$, as concentrações deste pigmento foram bem inferiores, apresentando inclusive tendência de correlação negativa entre as variáveis. Segundo dados de eutrofização do Portal Hidrológico do Ceará, a classificação do reservatório Pacajus variou de oligotrófico à eutrófico nesse período em estudo, sendo as 5 últimas classificações registradas como mesotrófico. Essa classificação pode refletir nas baixas concentrações de clorofila $a$ e na contagem de cianobactérias.

\section{CONCLUSÕES}

A deteminação e quantificação das cianobactérias se fazem necessárias para que ações de controle e minimização do crescimento desses organismos sejam implantadas, no intuito de reduzir a liberação de toxinas pelas cianobactérias, comprometendo a qualidade das águas.

As equações da reta, juntamente com os coeficientes de determinação apresentados, mostram que é válida a utilização delas para a quantificação do pigmento ficocianina nas águas de reservatórios, principalmente pela técnica de fluorimetria.

Devido à elevada abundância de cianobactérias nos reservatórios (grupo predominante - mais de $90 \%$ ) e os valores de correlações apresentadas (classificação forte/muito forte), a quantificação do pigmento está majoritariamente associada à presença das cianobactérias, indicando confiabilidade na estimativa desses microrganismos pela concentração da ficocianina.

Para clorofila $a$, não foram significativas as correlações encontradas, decorrente de outros fatores ambientais que não foram analisados neste trabalho, como a luminosidade e o $\mathrm{pH}$, por exemplo, e da não-exclusividade desse pigmento com as cianobactérias. Essas informações sugerem que esse pigmento não seja utilizado na estimativa da densidade de cianobactérias, sendo preferível o uso da ficocianina nessas estimativas.

\section{AGRADECIMENTOS}

O presente trabalho foi realizado com apoio da Coordenação de Aperfeiçoamento de Pessoal de Nível Superior - Brasil (CAPES) 
- Código de Financiamento 001 (PROEX 23038.000509/2020-82).

Agradecimento à COGERH por disponibilizar as amostras deste estudo, como também os resultados de cianobactérias realizados pelo ITEP.

\section{REFERÊNCIAS}

1. Grossman, A. R.; Schaefer, M. R., Chiang, G. G., Collier, J. L.; Microbiol. Rev. 1993, 57, 725 .

2. Román, R. B.; Alvarez-Pez, J. M.; Fernández, F. A.; Grima E. M.; J. Biotechnol. 2002, 93, 73.

3. Galland-Irmouli, A. V.; Pons, L.; Lucon, M.; Villaume, C.; Mrabet, N. T.; Guéant, J. L.; Fleurence, J.; J. Chromatogr. B 2000, 739, 117.

4. Nair, D.; Krishna, J. G.; Panikkar, M. V. N.; Nair, B. G.; Pai, J. G.; Nair, S. S.; Int. J. Biol. Macromol. 2018, 114, 679.

5. Parmar, A.; Singh, N. K.; Kaushal, A.; Sonawala, S.; Madamwar, D.; Bioresour. Technol. 2011, 102, 1795.

6. Saluri, M.; Kaldmäe, M.; Tuvikene, R.; Algal Res. 2019, 37, 115.

7. Patel, A.; Mishra, S.; Pawar, R.; Ghosh, P. K.; Protein Expression Purif. 2005, 40, 248.

8. Gregor, J.; Marsálek, B.; Sípková, H.; Water Res. 2007, 41, 228.

9. Padisák, J.; Chorus, I.; Welker, M.; Maršálek, B.; Kurmayer, R. Em Toxic cyanobacteria in water: a guide to their public health consequences, monitoring and management; Chorus, I., Welker, M., eds.; CRC Press: Flórida, 2021, cap. 13.

10. Mankiewicz, J.; Tarczynska, M.; Walter, Z.; Zalewski, M.; Acta Biol. Cracov., Ser. Bot. 2003, 45, 9.

11. Panosso, R.; Costa, I. A.; de Souza, N. R.; Attayde, J. L.; Oecologia Brasiliensis 2007, 59072, 970.

12. Kutser, T.; Limnol. Oceanogr. 2004, 49, 6.

13. Izydorczyk, K., Carpentier, C., Mrówczyński, J., Wagenvoort, A., Jurczak, T., Tarczyńska, M.; Water Res. 2009, 43, 4.

14. Pan, X., Wong, G. T., Ho, T. Y., Shiah, F. K., Liu, H.; Remote Sensing of Environment 2013, 128, 162.

15. Chaffin, J. D., Kane, D. D., Stanislawczyk, K., Parker, E. M.; Environ. Sci. Pollut. Res. 2018, 25, 25.

16. Kannaujiya, V. K., Sundaram, S., Sinha, R. P.; Phycobiliproteins: recent developments and future applications, Springer: Singapura, 2017.

17. Romay, C. H.; Gonzalez, R.; Ledon, N.; Remirez, D.; Rimbau, V.; Curr Protein Pept. Sci. 2003, 4, 207.

18. Santos, M. C. S.; Noyola, T. P.; Ramírez, R. O.; López, J. O.; Villanueva, R. O. C.; Process Biochem. 2004, 39, 2047.

19. Patil, G.; Raghavarao, K. S. M. S.; Biochem. Eng. J. 2007, 34, 156.

20. Vonshak, A. Em Spirulina Platensis Arthrospira: Physiology, CellBiology And Biotechnology; Vonshak, A., ed.; CRC Press: Great Britain, 1997.

21. Horváth, H.; Kovács, A. W.; Riddick, C.; Présing, M.; Eur. J. Phycol. 2013, 48, 278.

22. Yacobi, Y. Z.; Köhler, J.; Leunert, F.; Gitelson, A.; Limnol. Oceanogr.: Methods 2015, 13, 157.

23. Viskari, P. J.; Colyer, C. L.; Anal. Biochem. 2003, 319, 263.

24. Boussiba, S.; Richmond, A. E.; Arch. Microbiol. 1979, 120, 155.
25. Soni, B.; Kalavadia, B.; Trivedi, U.; Madamwar, D.; Process Biochem. 2006, 41, 2017.

26. Bennett, A.; Bogorad, L.; J. Cell Biol. 1973, 58, 419.

27. Lawrenz, E.; Fedewa, E. J.; Richardson, T. L.; J. Appl. Phycol. 2011, 23, 865 .

28. Sarada, R. M. G. P.; Pillai, M. G.; Ravishankar, G. A.; Process Biochem. 1999, 34, 795.

29. Silveira, S. T.; Burkert, J. F. D. M.; Costa, J. A. V.; Burkert, C. A. V.; Kalil, S. J.; Bioresour. Technol. 2007, 98, 1629.

30. Tsola, S. L., Renta, C., Macingo, S. C., Karayanni, H.; Oceanol. Hydrobiol. Stud. 2018, 47, 118.

31. Lehman, E. M.; Water Res. 2007, 41, 795.

32. Pavlac, M. M.; Smith, T. T.; Thomas, S. P.; Makarewicz, J. C.; Edwards, W. J.; Pennuto, C. M.; Boyer, G. L.; J. Great Lakes Res. 2012, 38, 78.

33. Seppälä, J.; Ylöstalo, P.; Kaitala, S.; Hällfors, S.; Raateoja, M.; Maunula, P.; Estuarine, Coastal and Shelf Sci. 2007, 73, 489.

34. Stewart, D. E.; Farmer, F. H.; Limnol. Oceanogr. 1984, 29, 392.

35. Thomson-Laing, G., Puddick, J., Wood, S. A.; Harmful Algae 2020, 97, 101869.

36. Yéprémian, C.; Catherine, A.; Bernard, C.; Congestri, R.; Elersek, T.; Pilkaityte, R. Em Handbook of cyanobacterial monitoring and cyanotoxin analysis; Meriluoto, J., Spoof, L., Codd, G. A., eds.; John Wiley \& Sons: Pondicherry, 2017, SOP 3

37. Araújo, S. M. S.; Rios Eletrônica 2011, 5, 5.

38. http://antigo.anvisa.gov.br/documents/10181/2721567/RDC_166_2017_ COMP.pdf/d5fb92b3-6c6b-4130-8670-4e3263763401, acessada em Agosto 2021.

39. APHA; Standard Methods for the Examination of Water and Wastewater; Baird, R. B., Eaton, A. D., Rice, E. W., eds.; $23^{\text {th }}$ ed.; Amer Public Health Assn: Washington, 2017.

40. UNESCO; Microscopic and molecular methods for quantitative phytoplanckton analysis; Karlson, B., Cusack, C., Bresnan, E., eds.; UNESCO: Paris, 2010.

41. Zhi, X.; Yuexin, S.; Jin, M.; Lujie, Z.; Zijian, D.; 13th IEEE International Conference on Electronic Measurement \& Instruments (ICEMI), Yangzhou, China, 2017.

42. Skoog, D. A.; Holler, F. J.; Crouch, S. R.; Princípios de Análise Instrumental, Editora Bookman: Porto Alegre, 2009.

43. Bennett, A.; Bogorad, L.; Biochemistry 1971, 10, 3625.

44. Yoshikawa, N.; Belay, A.; J. AOAC Int. 2008, 3, 91.

45. Barber, D. J. W.; Richards, J. T.; Photochem. Photobiol. 1977, 6, 25.

46. Brient, L., Lengronne, M., Bertrand, E., Rolland, D., Sipel, A., Steinmann, D., Baudin, I., Legeas, M., Le Rouzic. B., Bormans, M.; J. Environ. Monit. 2008, 10, 248.

47. http://conama.mma.gov.br/?option=com_sisconama\&task=arquivo download\&id=450, acessada em Agosto 2021.

48. Streit, N. M.; Canterle, L. P.; do Canto, M. W.; Hecktheuer, L. H. H. Cienc. Rural. 2005, 35, 748.

49. Paerl, H.W.; Justic, D. Em Estuarine Ecology; Day, J. W., Crump, B. C., Kemp, W. M., Yáñez-Arancibia, A., eds.; Wiley-Blackwell: New Jersey, 2013, cap. 4. 